\title{
Ecologia de duas espécies de Pachyurus (Perciformes, Sciaenidae) do rio Tocantins, na região represada pela UHE Serra da Mesa, Goiás
}

\author{
Andreza C. G. Pacheco ${ }^{1}$, Míriam P. Albrecht ${ }^{1} \&$ Érica P. Caramaschi ${ }^{1,2}$ \\ 1. Laboratório de Ecologia de Peixes, Departamento de Ecologia, IB, CCS, Universidade Federal do Rio de Janeiro, CP 68020, Av. Pau \\ Brasil 211, Ilha do Fundão, 21941-590 Rio de Janeiro, RJ. (andrezagomes@ gmail.com) \\ 2. Bolsista de Produtividade CNPq.

\begin{abstract}
Ecology of two species of Pachyurus (Perciformes, Sciaenidae) from the Tocantins River, in the region impounded by the Serra da Mesa hydroelectric dam, Goiás. Pachyurus schomburgkii Günther, 1860 and P. paucirastrus Aguilera, 1983 occur in the Tocantins-Araguaia basin, where the latter is endemic. The species were found in the upper Tocantins, in the region where it was impounded by the Serra da Mesa hydroelectric dam. Fourteen sites were sampled but both species were restricted to two sites (FU and PG) located downstream from the dam. Fishes were bimonthly captured from December 1995 to December 2002 with standardized gillnets. We present data on the distribution, diet, physiological condition and the reproductive status of these species, and focus on the alterations related to the impoundment. Most specimens were adult, and the number of reproductive individuals decreased after damming. Species were classified as benthic invertivores, feeding mainly on immature insects. Niche breadth was significantly higher to P. paucirastrus, and, for both species, during the pre-impoundment phase. Interspecific diet overlap was high; however, resource partitioning was more pronounced in the site immediately downstream from the dam (FU) than partitioning in the more distant site (PG). Values relative to the condition factor indicated that $P$. schomburgkii must have profited more effectively from the available resources after the environmental changes than $P$. paucirastrus. Thus, factors related to feeding might have contributed to the disappearance of this species after the beginning of power plant operation. Pachyurus schomburgkii seems to have had a better adjustment to changes, and was more persistent in the area after the damming; however, it remained restricted to the more distant site, where it had been occasionally captured until it was no longer found, approximately four years after dam closure. Considering that many impoundments are planned to the rio Tocantins, we recommend further studies about $P$. paucirastrus in other stretches of the basin, in order to evaluate the conservation status of this species.
\end{abstract}

KEY WORDS. Pachyurus, biology, diet, impoundment, Tocantins River.

RESUMO. Pachyurus schomburgkii Günther, 1860 e P. paucirastrus Aguilera, 1983 ocorrem na bacia Tocantins-Araguaia, sendo a segunda endêmica da bacia. Ambas foram encontradas na região do alto Tocantins, onde este foi represado pela usina hidrelétrica Serra da Mesa, tendo sido capturadas somente em dois de 14 pontos amostrados, sendo ambos (FU e PG) a jusante da barragem. Os peixes foram amostrados bimestralmente com redes de espera padronizadas entre dezembro de 1995 e dezembro de 2002. São apresentados dados de distribuição, dieta, condição fisiológica e estado reprodutivo das duas espécies, focando possíveis modificações em decorrência da instalação da barragem. A maioria dos indivíduos capturados eram adultos e o número de reprodutivos diminuiu após o represamento. As duas espécies foram classificadas como invertívoras bentônicas, alimentando-se principalmente de formas imaturas de insetos. A amplitude de nicho foi significativamente maior para P. paucirastrus, e maior na fase anterior ao represamento, para ambas as espécies. A sobreposição interespecífica da dieta foi alta, porém, a partilha de recursos foi maior no ponto imediatamente abaixo da represa (FU) do que naquele mais distante (PG). Os valores obtidos para o fator de condição indicaram que $P$. schomburgkii deve ter utilizado mais efetivamente os recursos disponíveis após as mudanças ambientais do que P. paucirastrus. Dessa forma, problemas relativos à alimentação podem ter contribuído para o desaparecimento dessa espécie durante a fase de operação da usina. Pachyurus schomburgkii parece ter se ajustado melhor às mudanças e mostrou-se mais persistente na área após o represamento, porém restrita ao ponto mais distal da barragem, tendo sido capturada de forma esporádica até desaparecer, aproximadamente quatro anos após a formação do reservatório. Considerando a série de usinas hidrelétricas projetadas para o rio Tocantins, recomendam-se estudos intensivos sobre P. paucirastrus em outras partes da bacia, de forma que seja avaliado seu real estado de conservação.

PALAVRAS-CHAVE. Pachyurus, biologia, dieta, represamento, rio Tocantins.

Historicamente, o desenvolvimento industrial e o crescimento populacional estão associados a um aumento na demanda de água e energia. Uma das formas de atender a este crescente consumo é através do represamento de rios (BRANDIMARTE et al., 1999). Entretanto, especialmente quando são feitas em série, as barragens quebram a conectividade montante-jusante, que é uma característica natural de rios (ARAúso-Lima et al., 1995), formando ambientes ecologicamente heterogêneos e complexos, cuja estrutura e dinâmica são intermediárias entre aquelas de um rio e um lago (MARGALEF, 1983).

Um efeito inevitável do represamento para a comunidade de peixes é a alteração na composição e abundância de espécies, com proliferação de algumas e redução ou até eliminação de outras (AgOSTiNHo, 1992; Agostinho et al., 1992; Agostinho et al., 1999), além de efeitos sobre a alimentação (HAHn et al., 1997).

Devido à rápida transformação na dinâmica da água, é esperada uma alteração na disponibilidade de recursos alimentares, que afetará as interações bióticas e levará os organismos a responderem de formas distintas às novas condições (HAHN et al., 1997). Neste contexto, o estudo da alimentação é uma importante ferramenta para avaliar as modificações que ocorrem em uma comunidade após um distúrbio como o represamento, podendo fornecer, ainda, importantes subsídios para o entendimento do funcionamento do ecossistema (HAHN et al., 1997). 
A porção superior do rio Tocantins foi represada pela instalação da usina hidrelétrica de Serra da Mesa em outubro de 1996. Foram registradas três espécies de cianídeos na região: Pachyurus schomburgkii Günther, 1860, Pachyurus paucirastrus Aguilera, 1983 e Plagioscion squamosissimus Heckel, 1840. Pachyurus paucirastus e $P$. schomburgkii foram relativamente pouco abundantes e restritas à área a jusante da barragem.

A família Sciaenidae inclui 78 gêneros e 287 espécies distribuídas em regiões tropicais e temperadas (CHAO, 1986). As espécies do grupo são conhecidas por corvinas ou pescadas e apresentam grande importância para a pesca marinha e continental. Entre os quatro gêneros de Sciaenidae de água doce endêmicos da América do Sul, Pachyurus é o que apresenta maior número de espécies (9) e maior área de ocorrência geográfica (CASATTI, 2003). Pachyurus schomburgkii encontra-se distribuída nas bacias dos rios Orinoco e Amazonas e P. paucirastrus é endêmica da Bacia Tocantins-Araguaia (CASATTI, 2003).

Estudos abordando a alimentação, aspectos populacionais e reprodutivos das espécies do gênero ainda são muito escassos. As espécies de Pachyurus já foram classificadas como insetívoras (MÉronA et al., 2001), bentívoras (CASATTI, 2002) ou piscívoras (Alvim \& Peret, 2004). Braga (1990) e Mérona et al. (2001) estudaram a variação na dieta de $P$. schomburgkii em dois outros trechos do rio Tocantins.

O presente trabalho teve como objetivo descrever a ecologia e distribuição de $P$. schomburgkii e $P$. paucirastrus no trecho superior do rio Tocantins na área de influência da usina hidrelétrica Serra da Mesa e analisar as possíveis modificações nesses aspectos em decorrência do represamento. Foram enfocados a composição qualitativa e quantitativa da dieta, a condição fisiológica e alguns aspectos reprodutivos das espécies, partindo da premissa de que o represamento constitui um distúrbio ao qual as espécies de peixes respondem de forma diferenciada em termos demográficos e biológicos.

\section{MATERIAL E MÉTODOS}

A barragem da UHE Serra da Mesa foi implantada em outubro de 1996 no trecho superior do rio Tocantins, município de Minaçu, a 1.790km de sua foz. O reservatório de Serra da Mesa é o maior do Brasil em volume de água, com 54,4 bilhões de $\mathrm{m}^{3}$ e o quinto maior em área alagada $\left(1.784 \mathrm{~km}^{2}\right)$ (DE FiLIPPO et al., 1999).

A área inicial de estudo está localizada entre as

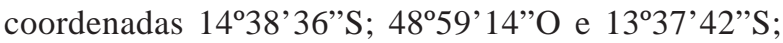
48 $07^{\circ}$ '08'O (mapa e detalhes em AlBReChT \& PELLEGRINICARAMASCHI, 2003) e compreendeu 14 pontos distribuídos em três áreas (Montante, Reservatório e Jusante). A Área de Montante, localizada acima do remanso do reservatório apresentou sempre características lóticas; a Área do Reservatório, localizada logo acima da barragem, apresentou variação na dinâmica hídrica (lótica versus lêntica) devido ao represamento; a Área de Jusante, localizada abaixo da barragem, apresentou características lóticas irregulares. A última compreendeu dois pontos: Funai (FU), localizado no trecho imediatamente a jusante e Porto Garimpo (PG), localizado a cerca de $40 \mathrm{~km}$ da barragem.
O material utilizado no presente estudo provém de amostragens planejadas para um estudo mais amplo, direcionadas ao monitoramento da ictiofauna, não tendo sido especialmente direcionadas às espécies de estudo. As coletas foram realizadas na fase anterior ao represamento (fase rio - dezembro/1995 a outubro/1996), durante o enchimento do reservatório (fase enchimento dezembro/1996 a abril/1998) e após o início da operação da usina (fase operação - junho/1998 a fevereiro/2000 e fase monitoramento - fevereiro/2001 a dezembro/2002). No presente trabalho, as fases enchimento e operação foram agrupadas como fase pós-represamento, para facilitar algumas análises e interpretação dos resultados. Espécimes-testemunho de $P$. paucirastrus e $P$. schomburgkii foram tombados no Museu Nacional do Rio de Janeiro (MNRJ-12557, 12601, 12672, 12740 e MNRJ15216, respectivamente).

Os exemplares de Pachyurus foram obtidos a partir de coletas realizadas bimestralmente com baterias de redes de espera de $15 \mathrm{~mm}$ a $150 \mathrm{~mm}$ entre-nós adjacentes, deixadas por um período de 24 horas em cada ponto e vistoriadas a cada 8 horas. Após cada vistoria, os peixes foram transportados em gelo para o laboratório de campo, onde foram obtidos: comprimento padrão $(\mathrm{Cp})$, peso total $(\mathrm{Pt})$, peso da gônada, classificação macroscópica do sexo e estágio de maturação gonadal. Os estômagos com conteúdo foram retirados e fixados em formol $5 \%$ para posterior análise.

Para avaliar e comparar a abundância e distribuição espaço-temporal das espécies, foi utilizada captura por unidade de esforço (CPUE), conforme a equação:

CPUEn $=\mathrm{N} /\left(\mathrm{m}^{2} * \mathrm{~h}\right)$, onde: $\mathrm{N}=$ número de indivíduos capturados, $\mathrm{m}^{2}$ = área das redes de emalhar, $\mathrm{h}$ = tempo de exposição das redes de emalhar. O denominador da equação, no período de estudos, foi padronizado em $650 \mathrm{~m}^{2}$.

Para avaliação da atividade reprodutiva, foram contabilizados os indivíduos reprodutivos, nãoreprodutivos e imaturos e calculada a proporção entre os sexos nas fases anterior e posterior ao represamento. $\mathrm{O}$ teste de qui-quadrado foi usado para testar a divergência da proporção esperada de 1:1 entre os sexos.

Para o estudo da dieta, foram analisados 71 estômagos de $P$. schomburgkii $(\mathrm{Cp}=11,0-35,3 \mathrm{~cm})$ e 61 estômagos de $P$. paucirastrus $(\mathrm{Cp}=10,0-23,5 \mathrm{~cm})$. Os conteúdos estomacais foram analisados em microscópio estereoscópico.

A análise quantitativa dos itens foi feita através do método volumétrico (HyNEs, 1950; HysLoP, 1980), com auxílio de uma placa de vidro e uma escala milimetrada acoplada, conforme descrito em AlBRECHT \& CARAMASCHI (2003). No caso dos itens grandes, o volume foi medido por deslocamento da coluna d'água em proveta milimetrada. Os itens alimentares foram identificados até a mais alta resolução taxonômica possível. Os dados obtidos através da quantificação do volume foram combinados com a freqüência de ocorrência de cada item, para obtenção do Índice Alimentar (IAi) proposto por KAWAKAMI \& VAZZOLER (1980), com o objetivo de distinguir a importância relativa de cada item em termos quantitativos. 
Foi também investigada a amplitude do nicho trófico, que pode ser referida como a diversidade de presas consumidas (MAGURRAN, 1988), avaliada através do índice de diversidade de Shannon (KREBS, 1989). Diferenças significativas entre as amplitudes de nicho foram verificadas através do teste de Hutcheson (ZAR, 1984).

A sobreposição alimentar é utilizada como uma medida de similaridade entre a dieta das espécies e foi calculada através do índice simétrico de Pianka (KREBS, 1989).

Alterações no estado nutricional dos indivíduos de $P$. paucirastrus e $P$. schomburgkii foram verificadas pelo fator de condição $(K)$, através da fórmula $K=W_{c} / L_{t}^{b}$ (LeCREN, 1951), onde $\mathrm{W}_{\mathrm{c}}=$ peso do corpo $(\mathrm{g})$ (subtraindo o peso das gônadas), $\mathrm{L}_{\mathrm{t}}=$ comprimento padrão $(\mathrm{cm}), \mathrm{b}=$ coeficiente de regressão $\mathrm{W}_{\mathrm{c}} / \mathrm{L}_{\mathrm{t}}$ (especíe-específico). Os valores foram comparados, para uma mesma espécie, entre as fases anterior e posterior à formação do reservatório através de um teste $t$ (ZAR, 1984).

\section{RESULTADOS}

Pachyurus paucirastrus, além de ocorrer em menor número de indivíduos, deixou de ser capturada a partir de um ano e meio após o início da operação comercial da usina, em 1998. Houve oscilações no número de indivíduos capturados a cada mês de coleta, com tendência à diminuição da fase rio ao monitoramento. Ambas as espécies foram mais abundantes no ponto PG (Figs. 1a,b), enquanto que no ponto FU não foram mais capturadas após o enchimento do reservatório. Posteriormente, durante o monitoramento (2001-2002),

a
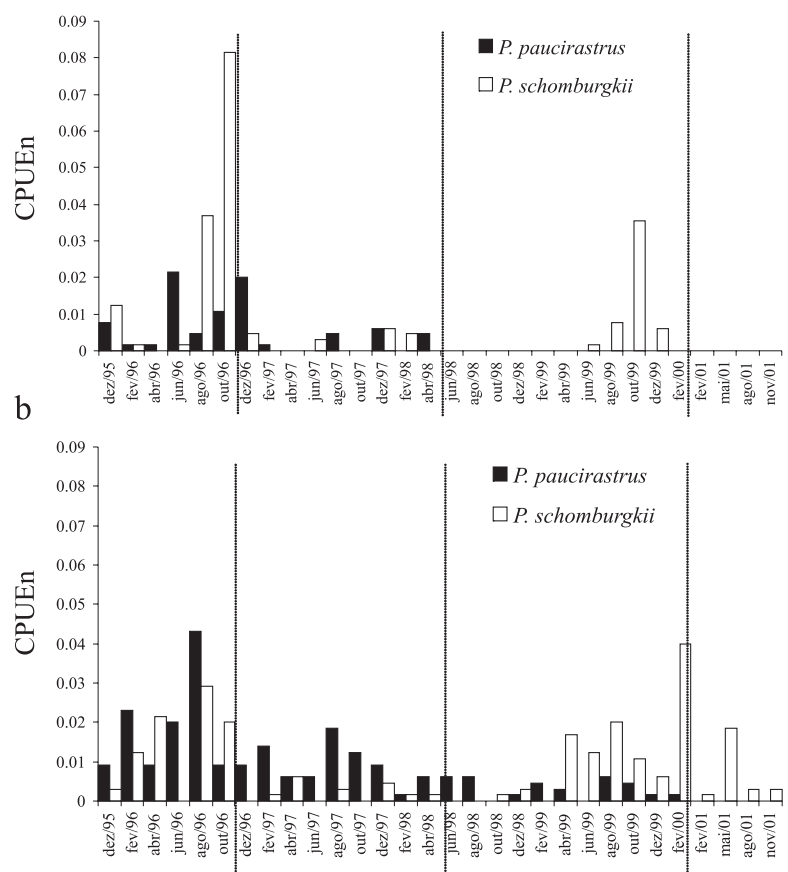

Fig. 1. Abundância medida pela Captura por Unidade de Esforço (CPUEn) para Pachyurus schomburgkii Günther, 1860 e P. paucirastrus Aguilera, 1983 no alto rio Tocantins, nas localidades Funai (a) e Porto Garimpo (b), a jusante da represa de Serra da Mesa, GO. As linhas verticais pontilhadas separam, em ordem cronológica, as fases rio (pré-represamento), enchimento, operação e monitoramento (pós-represamento).
P. paucirastrus deixou de ser capturado também em PG (desapareceu da área estudada).

A amplitude de comprimento padrão foi maior para $P$. paucirastrus $(8,1$ a $38,8 \mathrm{~cm})$ do que para $P$. schomburgkii (9,7 a $35,3 \mathrm{~cm})$, porém esta espécie apresentou média de tamanho maior $(13 \mathrm{~cm}$ e $22 \mathrm{~cm}$, respectivamente). A variação no peso também foi maior para $P$. paucirastrus (13,4 e 956,1g) do que para P. schomburgkii (16 e 487,3g), sendo o peso médio de $P$. schomburgkii $(207 \mathrm{~g})$ maior que de $P$. paucirastrus $(50 \mathrm{~g})$.

A proporção entre os sexos de indivíduos adultos das populações de $P$. paucirastrus e $P$. schomburgkii foi de aproximadamente 1 macho para 2 fêmeas e 1 macho para 1 fêmea, respectivamente, nas fases anterior e posterior ao represamento, tendo sido significativamente diferente em $P$. paucirastrus $\left(\chi^{2}=21,64, P<0.01\right)$. A maior parte dos indivíduos adultos das duas espécies se encontrava em estádios não reprodutivos e raramente foram capturados indivíduos imaturos. O número de reprodutivos de ambos os sexos diminuiu para $P$. paucirastrus após o represamento. Para $P$. schomburgkii, o número de machos reprodutivos e fêmeas não reprodutivas aumentou. Para esta espécie, o número de indivíduos reprodutivos capturados foi inferior à metade do número de não reprodutivos (Tab. I).

Considerando a dieta das espécies como um todo, o item alimentar mais importante foi ninfa de Polymitarcyidae (Ephemeroptera) para ambas as espécies (Figs. 2a,b). Larva de Chironomidae (Diptera) foi o segundo item mais importante na alimentação de $P$. paucirastrus e o quarto para $P$. schomburgkii. O segundo item mais importante de $P$. schomburgkii foi Oligochaeta. $\mathrm{O}$ item matéria orgânica + sedimento $(\mathrm{MO}+\mathrm{S})$ foi o terceiro mais consumido pelas duas espécies.

Modificações temporais na dieta foram observadas (Tab. II). As análises foram feitas separadamente por ponto de coleta nas fases anterior e posterior ao represamento, com exceção de $P$. paucirastrus em FU, que apresentou número muito reduzido de indivíduos, não possibilitando a separação temporal.

$\mathrm{Na}$ fase anterior ao represamento, ninfa de Polymitarcyidae foi o principal item consumido por $P$. paucirastrus em PG e por $P$. schomburgkii em FU e PG (Tab. II). Após a formação do reservatório, o principal item consumido por $P$. paucirastrus em PG e por $P$. schomburgkii em FU passou a ser larva de Chironomidae, seguido por $\mathrm{MO}+\mathrm{S}$. Os itens mais consumidos por $P$. schomburgkii em PG na fase posterior à formação do reservatório foram $\mathrm{MO}+\mathrm{S}$ e Oligochaeta (Tab. II).

Tabela I. Proporção sexual (PS, M:F), número de machos e fêmeas não reprodutivos (MnR e FnR), reprodutivos (MR e FR), e de indivíduos imaturos (I) de Pachyurus schomburgkii Günther, 1860 e P. paucirastrus Aguilera, 1983 nas localidades a jusante da barragem de Serra da Mesa, alto rio Tocantins (GO), nas fases pré e pós represamento.

\begin{tabular}{lccccccc}
\hline \multicolumn{7}{c}{$P$. paucirastrus } \\
\hline & MnR & FnR & MR & FR & I & PS & total \\
pré & 15 & 35 & 12 & 28 & 3 & $1: 2,3^{*}$ & 93 \\
pós & 17 & 28 & 8 & 20 & 2 & $1: 1,92^{*}$ & 75 \\
\hline \multicolumn{7}{c}{$P$. schomburgkii } \\
\hline & MnR & FnR & MR & FR & I & PS & total \\
pré & 56 & 40 & 22 & 18 & 2 & $1,3: 1$ & 138 \\
pós & 39 & 50 & 27 & 10 & 1 & $1,1: 1$ & 127 \\
\hline
\end{tabular}


Tabela II. Freqüência de Ocorrência (FO\%), Volume Relativo (VO\%) e Índice Alimentar (IAi) dos itens consumidos por Pachyurus schomburgkii Günther, 1860 e P. paucirastrus Aguilera, 1983 no alto rio Tocantins (GO) nas localidades Funai (FU) e Porto Garimpo (PG) a jusante da barragem de Serra da Mesa, durante as fases anterior e posterior ao represamento. Os asteriscos indicam valores menores que 0,01.

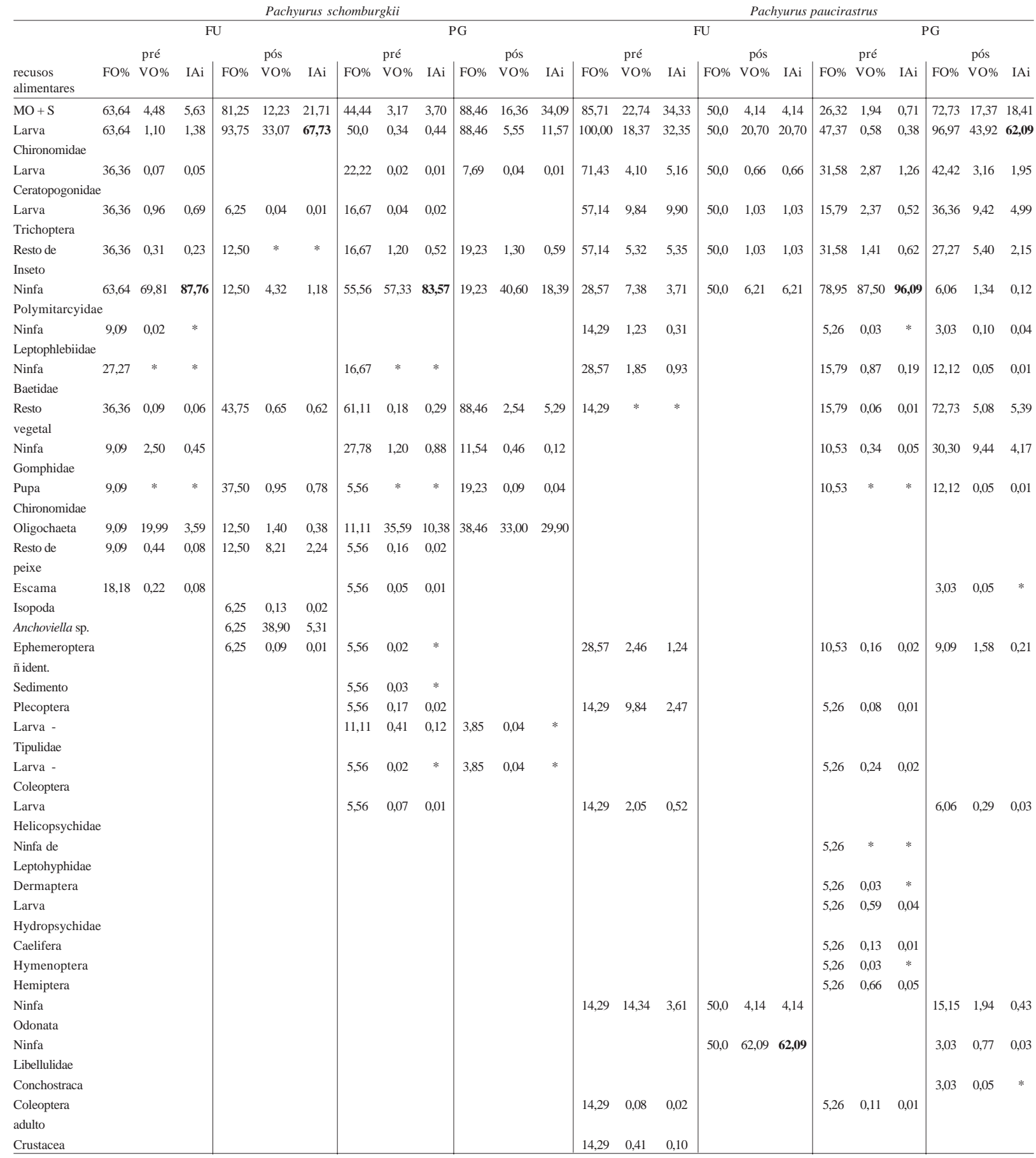

A amplitude de nicho trófico foi significativamente maior $(\mathrm{t}=2,58 ; \mathrm{p}<0,05)$ para $P$. paucirastrus considerando-se os dois pontos em conjunto $(P$. paucirastrus: $\mathrm{H}^{\prime}=1,14 ;$ P. schomburgkii: $\left.\mathrm{H}^{\prime}=1,04\right)$. Na comparação entre pontos, a maior amplitude para $P$. paucirastrus ocorreu em PG e para $P$. schomburgkii em FU. Foram observadas maior amplitude de nicho e maior riqueza de itens alimentares na fase anterior ao represamento. Esse padrão foi verificado para ambas as espécies em PG (Tab. III). Para P. schomburgkii, a amplitude de nicho foi maior na fase pré-represamento em ambos os pontos (Tab. IV), sendo essa diferença significativa ( $P$. paucirastrus em $\mathrm{PG} ; t=2,09 ; P$. schomburgkii em PG; $t=4,92$ e FU $t=2,65$; p < 0,05).

Foi registrada alta sobreposição de nicho entre as duas espécies, considerando os dois pontos em conjunto $(\theta=0,85)$. Os valores de similaridade intraespecífica da dieta foram altos para $P$. schomburgkii entre os pontos FU e PG $(\theta=0,89)$, ao contrário de $P$. paucirastrus $(\theta=0,42)$. Em PG, as espécies apresentaram dietas mais similares 
Tabela III. Riqueza de itens alimentares (S), amplitude de nicho $\left(H^{\prime}\right)$, sobreposição alimentar $(\theta)$ e número de estômagos analisados (n), para Pachyurus schomburgkii Günther, 1860 e $P$. paucirastrus Aguilera, 1983 em Porto Garimpo (PG) nas fases anterior (pré) e posterior (pós) ao represamento do alto rio Tocantins, e nas duas fases (total). Os asteriscos indicam valores significativamente diferentes entre si ( $P$. paucirastrus $t=2,09 ; P$. schomburgkii $t=4,92 ; \alpha=0,05)$.

\begin{tabular}{|c|c|c|c|c|c|c|}
\hline & \multicolumn{6}{|c|}{ Porto Garimpo } \\
\hline & \multicolumn{3}{|c|}{ P. paucirastrus } & \multicolumn{3}{|c|}{ P. schomburgkii } \\
\hline & pré & pós & total & total & pré & pós \\
\hline $\bar{S}$ & 22 & 14 & 27 & 19 & 19 & 11 \\
\hline $\mathrm{H}^{\prime}$ & $1.15^{*}$ & $1.01 *$ & 1.13 & 1.01 & $1.12 *$ & $0.85 *$ \\
\hline$\theta$ & \multicolumn{2}{|c|}{0.05} & \multicolumn{2}{|c|}{0.81} & \multicolumn{2}{|c|}{0.95} \\
\hline$n$ & 19 & 33 & 52 & 44 & 18 & 26 \\
\hline
\end{tabular}

a
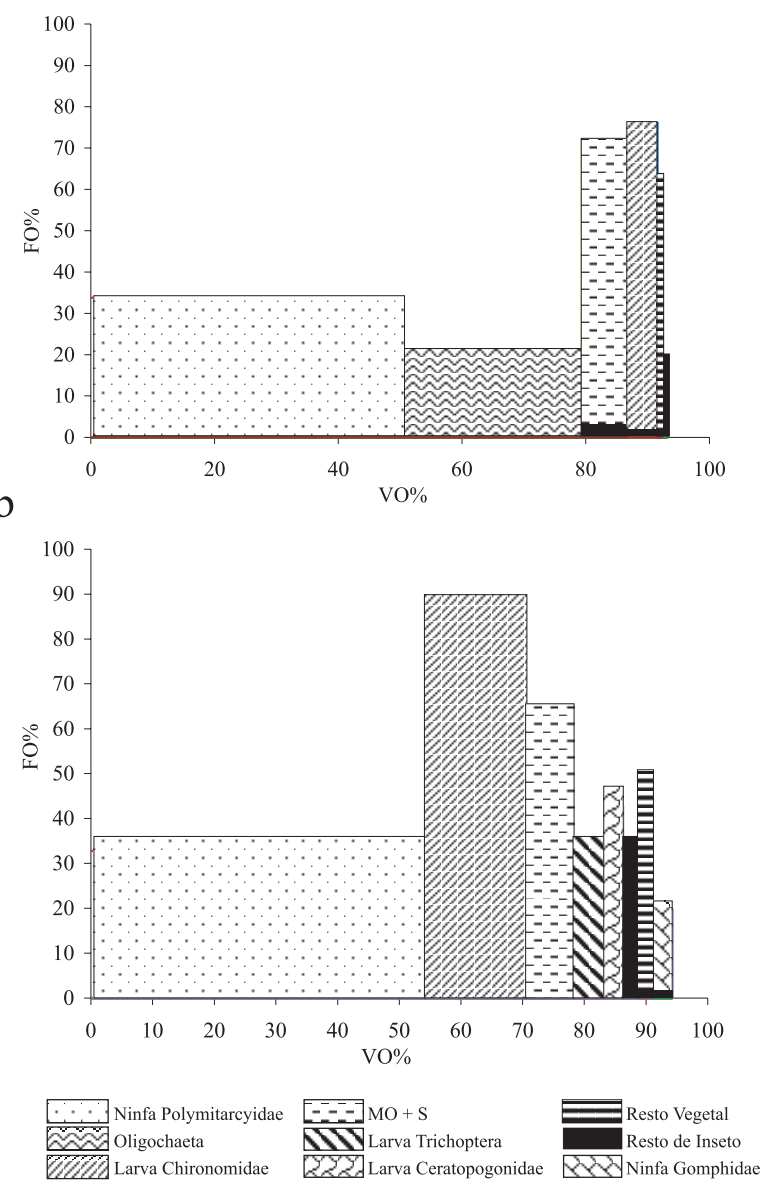

Fig. 2. Representação gráfica do volume $(\mathrm{VO} \%)$ e freqüência de ocorrência $(\mathrm{FO} \%)$, combinados no Índice Alimentar (IAi) dos principais itens consumidos por (a) Pachyurus schomburgkii Günther, 1860 e (b) P. paucirastrus Aguilera, 1983.

entre si do que em FU (Tabs. III e IV). A sobreposição alimentar entre as fases anterior e posterior ao represamento foi baixa para $P$. paucirastrus em $\mathrm{PG}$ e para $P$. schomburgkii em FU. A última, porém, apresentou alta similaridade entre as duas fases no ponto PG (Tab. III).

Os valores do fator de condição médio para as duas espécies foram semelhantes (Tab. V). O fator de condição de $P$. paucirastrus foi significativamente menor durante a fase pós-represamento, ao passo que o de $P$. schomburgkii foi significativamente maior $(\mathrm{p}<0,05)$.
Tabela IV. Riqueza de itens alimentares (S), amplitude de nicho $\left(\mathrm{H}^{\prime}\right)$, sobreposição alimentar $(\theta)$ e número de estômagos analisados (n), para Pachyurus schomburgkii Günther, 1860 e P. paucirastrus Aguilera, 1983 na localidade Funai (FU) nas fases anterior (pré) e posterior (pós) ao represamento e nas duas fases (total). Os asteriscos indicam valores que foram significativamente diferentes entre si $(t=2,65 ; \alpha=0,05)$.

\begin{tabular}{|c|c|c|c|c|}
\hline & & Funai & & \\
\hline & P. pauciras & & hombu & \\
\hline & total & total & pré & pós \\
\hline$S$ & 16 & 17 & 14 & 12 \\
\hline $\mathrm{H}^{\prime}$ & 1.08 & 1.03 & $1.05 *$ & $0.87 *$ \\
\hline$\theta$ & & & & \\
\hline$n$ & 9 & 27 & 11 & 16 \\
\hline
\end{tabular}

Tabela V. Fator de condição (K) de Pachyurus schomburgkii Günther, 1860 e $P$. paucirastrus Aguilera, 1983 nas fases pré e pósrepresamento, número $(n)$ e amplitude de tamanho (Lp mín-máx) dos exemplares considerados na análise. Asteriscos indicam valores de K significativamente diferentes entre as fases pré e pósrepresamento para uma mesma espécie.

\begin{tabular}{lcccccc}
\hline & \multicolumn{3}{c}{$P$. paucirastrus } & \multicolumn{3}{c}{$P$ schomburgkii } \\
& total & pré & pós & total & pré & Pós \\
\hline K médio & 0.025 & $0.031^{*}$ & $0.019^{*}$ & 0.02 & $0.018^{*}$ & $0.028^{*}$ \\
$n$ & 155 & 79 & 76 & 248 & 131 & 117 \\
Lp min- & $10.0-$ & $10.6-$ & $10.0-$ & $9.7-$ & $9.7-$ & $11.2-$ \\
máx & 23.8 & 23.8 & 21.5 & 35.3 & 34.0 & 35.3 \\
\hline
\end{tabular}

\section{DISCUSSÃO}

As duas espécies de Pachyurus foram relativamente pouco abundantes e ocorreram somente a jusante do reservatório de Serra da Mesa, ao contrário do outro cianídeo, $P$. squamosissimus, bastante abundante e bem distribuído nos trechos amostrados do alto Tocantins (montante, reservatório e jusante) (Rosana Mazzoni, com. pess.). Os túneis de desvio, abertos por ocasião da construção da represa (iniciada em 1988 e interrompida até outubro de 1995), eram longos e sem anteparos, provavelmente não oferecendo refúgio a fracos nadadores. Assim, podem ter representado uma barreira para o deslocamento dessas espécies para montante, tendo restringido as populações aos pontos abaixo da barragem, mesmo antes do seu fechamento. A distribuição dessas espécies anteriormente à construção dos túneis, entretanto, não é conhecida.

As modificações no ambiente fluvial que sucedem o represamento são diferentes a jusante em comparação com a área do reservatório. Entre os efeitos a jusante, estão aqueles decorrentes das alterações no fluxo e na qualidade da água evertida (Agostinho et al., 1992). Em Serra da Mesa, com a interrupção do fluxo do rio durante a fase de enchimento do reservatório, formaram-se poções a jusante da barragem nos quais o aumento da densidade de peixes pode ter levado ao aumento de interações de predação. Essa pode ter sido uma das causas da redução da captura de indivíduos de Pachyurus no ponto imediatamente abaixo da barragem. O consumo de cianídeos a jusante foi registrado no conteúdo estomacal de Plagioscion squamosissimus após o represamento (Andreza C. G. Pacheco, dados não publicados).

Nos novos ambientes decorrentes de represamentos, algumas espécies são favorecidas e 
proliferam, enquanto outras tendem à extinção local (HAHN et al., 1998) ou se deslocam à procura de condições mais favoráveis (Agostinho et al., 1999). Este parece ser o caso das duas espécies de Pachyurus no ponto imediatamente abaixo da barragem (FU), e de $P$. paucirastrus mesmo no ponto mais distante (PG). Em janeiro de 2001 o rio Tocantins foi novamente barrado para a formação do reservatório da UHE de Cana Brava, o que transformou a área a jusante de Serra da Mesa em um novo ambiente lêntico. CASATti (2003) afirma que Pachyurus e Pachypops são gêneros característicos de lagos, porém, nenhuma das duas espécies de Pachyurus ocupou a área de reservatório, tendo o último registro de P. schomburgkii ocorrido em novembro de 2001. Essas espécies não eram muito abundantes neste trecho do rio Tocantins, mesmo antes da formação do primeiro reservatório. Porém, o desaparecimento de $P$. paucirastrus ainda na fase enchimento e de $P$. schomburgkii no final da fase de operação de Serra da Mesa sugere que essas espécies não encontraram condições favoráveis e se deslocaram para locais mais a jusante, para afluentes laterais, ou foram localmente extintas no trecho confinado entre as duas barragens.

A grande maioria dos indivíduos capturados era de adultos e como indivíduos podem maturar em tamanhos pequenos não capturáveis pela rede de $15 \mathrm{~mm}$, não é possível estabelecer se esse dado reflete a estrutura da população ou a seletividade das redes. O desvio da proporção de 1:1 entre os sexos observado somente para $P$. paucirastrus pode dever-se a diferenças nas taxas de crescimento entre machos e fêmeas, que poderiam tornar um dos sexos mais vulnerável à predação (NIKOLSKI, 1963). A variação no número de indivíduos reprodutivos não demonstrou padrão claro; entretanto, a diminuição após o represamento sugere condições inadequadas à reprodução. Um estudo mais detalhado sobre estratégias reprodutivas seria desejável para a compreensão das variações populacionais das duas espécies.

A composição da dieta das duas espécies de Pachyurus permite classificá-las como invertívoras bentônicas, alimentando-se principalmente de formas imaturas de insetos associadas ao substrato de fundo. Esse hábito alimentar é favorecido pela posição da boca, que é pequena e sub-terminal em $P$. schomburgkii e inferior em $P$. paucirastrus (CASATTI, 2001). No trecho inferior do rio Tocantins, a jusante do reservatório de Tucuruí, foi reportado que $P$. schomburgkii se alimentou principalmente de invertebrados aquáticos $(70 \%)$ e terrestres $(30 \%)$ no período anterior ao represamento e, após, exclusivamente de invertebrados aquáticos (Mérona et al., 2001). Por outro lado, no trecho médioinferior do mesmo rio, a espécie foi considerada piscívora por Braga (1990). Outras espécies do gênero foram classificadas como piscívoras ou com tendência à piscivoria, como Pachyurus francisci (Cuvier, 1830) e $P$. squamipinnis Agassiz, 1831 no reservatório de Três Marias, alto rio São Francisco (Alvim \& Peret, 2004). Sobre a alimentação de $P$. paucirastrus, não existem informações prévias.

$\mathrm{Na}$ fase rio, ninfas de Ephemeroptera (Polymitarcyidae) foi destacadamente o item alimentar mais importante; entretanto, sua importância se restringiu à coleta de agosto de 1996, quando foram consumidas ninfas em estádio avançado de desenvolvimento. Os ovos presentes no abdômen e as tecas alares quase totalmente formadas indicam a proximidade do período de revoada reprodutiva desses insetos (MCCAFFERTY, 1998). Já que saem das galerias fabricadas no substrato para emergirem (PEREIRA \& DA-SiLVA, 1991), as ninfas se tornam mais disponíveis a predadores nessa ocasião (Jorge L. Nessimian, com. pess.). Isso demonstra oportunismo dos peixes, pois naquele momento esse foi um recurso extremamente abundante, tendo sido consumido inclusive por $P$. squamosissimus, espécie essencialmente piscívora (Andreza C. G. Pacheco, dados não publicados).

O consumo de ninfas de Polymitarcyidae foi reduzido após o represamento. A interrupção do fluxo d'água dessecou parte do substrato e alterou a oxigenação da água, o que pode ter provocado a migração de fêmeas para áreas mais favoráveis durante a revoada, ou mesmo diminuído a disponibilidade de alimentos para as ninfas. As mudanças na composição da dieta das duas espécies de Pachyurus após o represamento podem representar alterações na composição e/ou abundância dos diferentes táxons da comunidade de invertebrados bentônicos. A ausência de estudos sobre a macrofauna bentônica do rio Tocantins na área do reservatório de Serra da Mesa não permite avaliar os impactos diretos decorrentes da formação do reservatório para o grupo. No entanto, Oligochaeta, seguido de Chironomidae, são citados como dominantes a jusante de reservatórios (e.g. DumnickA, 1996). Considerando que a dieta dos peixes funciona como um amostrador indireto das alterações de composição e abundância de suas presas, a dominância desses organismos foi corroborada na área a jusante de Serra da Mesa. Na fase posterior ao represamento, Oligochaeta foi muito consumido por P. schomburgkii em PG e foi verificado um aumento na importância de larvas de Chironomidae e de sedimento + matéria orgânica $(\mathrm{MO}+\mathrm{S})$ para ambas as espécies. $\mathrm{MO}+\mathrm{S}$, neste caso, pode ser composta por restos digeridos de Oligochaeta e larva de Chironomidae e seus conteúdos, ou terem sido ingeridos junto com os insetos bentônicos. As alterações ambientais a jusante podem ter acarretado também uma substituição de espécies de Chironomidae, como registrado por KIKUCHI \& UIEDA (1998) ao avaliar tipos de hábitats com diferentes características de fluxo. Aqueles grupos que conseguiram permanecer ou que colonizaram esse ambiente após o represamento e se tornaram abundantes, foram intensamente predados. Os quironomídeos são usualmente os componentes mais abundantes e diversificados da comunidade de macroinvertebrados bentônicos (STRIXINO \& TRIVINHOSTRIXINO, 1998) e desempenham um importante papel na dieta de várias outras espécies encontradas no Tocantins (e. g. Leporinus taeniofasciatus Britski, 1997 - AlBRECHT \& Pellegrini-Caramaschi, 2003).

O elevado consumo de efemerópteros na dieta total das espécies acarretou o alto valor de sobreposição alimentar verificado. Entretanto, algumas diferenças espaciais e temporais foram observadas. A partilha foi maior no ponto mais próximo da represa (FU) do que no distal (PG). Em comunidades aquáticas, a segregação 
trófica é considerada mais importante que a separação de hábitats (Ross, 1986), porém variável de acordo com as condições ambientais que alteram a disponibilidade de recursos (e. g. sazonalidade - WinEMILLER, 1989). O grau de parentesco entre espécies também tem efeito significativo sobre a segregação ecológica (Ross, 1986); assim, é esperado que espécies congenéricas apresentem uma maior partilha de recursos, como observado para as duas espécies de Pachyurus em FU.

Alterações no comportamento alimentar são esperadas em ambientes represados e decorrem de mudanças na disponibilidade de recursos e também da plasticidade alimentar das espécies frente aos novos recursos (HAHN et al., 1997). A similaridade intraespecífica da dieta entre as fases foi muito baixa para $P$. paucirastrus em PG, indicando que esta foi bastante modificada após o represamento, quando a espécie passou a consumir uma maior diversidade de presas. O oposto foi demonstrado por $P$. schomburgkii em PG que, apesar da diversidade de itens consumidos ter sido reduzida, não substituiu seus principais recursos alimentares. No entanto, próximo à barragem, em um ambiente reduzido a poções (FU), P. schomburgkii alterou bastante sua dieta. Tais relações sugerem consumo mais eficiente dos recursos abundantes por $P$. schomburgkii, cabendo a $P$. paucirastrus ajustar-se aos recursos menos nutritivos ou de mais difícil obtenção.

Mesmo apresentando espectro alimentar menor, $P$. schomburgkii foi eficaz no uso dos novos recursos. O aumento no fator de condição de $P$. schomburgkii indica que esta espécie utilizou mais efetivamente os recursos disponíveis após as mudanças ambientais, como larva de Chironomidae e Oligochaeta, acumulando reservas corporais. Entretanto, essas reservas aparentemente não foram convertidas em esforço de reprodução, uma vez que a proporção de indivíduos reprodutivos diminuiu para ambas as espécies, especialmente para $P$. schomburgkii. Ao contrário, a redução significativa no fator de condição médio de $P$. paucirastrus após o represamento sugere que a alimentação foi um fator que contribuiu para o desaparecimento da espécie na área de estudo. Pachyurus schomburgkii mostrou-se mais persistente, pelo menos no ponto mais distante (PG), onde os efeitos do barramento podem ter sido minimizados pela distância da barragem e pela vazão de vários afluentes pequenos e do rio Preto, um afluente de porte médio.

Não são conhecidas com precisão a distribuição nem as áreas mais favoráveis à ocorrência dessas espécies, especialmente para a endêmica $P$. paucirastrus. Os dados aqui apresentados são os primeiros sobre a ecologia dessa espécie e esta mostrou-se prejudicada ou mais prontamente deslocada da área de influência do represamento. Considerando que outros reservatórios vêm sendo projetados para o rio Tocantins, recomendamse estudos intensivos em toda a bacia, de forma a reunir informações que permitam avaliar o real estado de conservação de $P$. paucirastrus.

Agradecimentos. Os convênios UFRJ/BIORIO/FurnasSerra da Mesa Energia S. A. e UFRJ/Fundação Educacional Charles Darwin/Furnas-CPFL financiaram os projetos "Estudos básicos sobre a ictiofauna do AHE Serra da Mesa" e "Monitoramento da ictiofauna de Serra da Mesa", respectivamente, dos quais o presente trabalho é oriundo. As autoras agradecem aos demais participantes do projeto pela obtenção dos dados de campo e laboratório, a Jorge L. Nessimian (Depto. Zoologia/UFRJ) pelo material bibliográfico e informações sobre insetos aquáticos e a Jean Valentin (Depto. Biologia Marinha/UFRJ), Marcelo F. G. Brito (PPGE/UFRJ) e Ricardo Iglesias Rios (Depto. Ecologia/UFRJ) pela revisão crítica da primeira versão do manuscrito. ACGP agradece ao CNPq (PIBICCNPq/UFRJ) pela bolsa de Iniciação Científica, MPA pela bolsa PDJ (proc. 151148/2005-8) e EPC pela bolsa PQ (proc. 309506/ 2003-4).

\section{REFERÊNCIAS BIBLIOGRÁFICAS}

Agostinho, A. A. 1992. Manejo de recursos pesqueiros em reservatórios In: Agostinho, A. A. \& Benedito-Cecílio, E. eds. Situação atual e perspectivas da ictiologia no Brasil. Documentos do IX Encontro Brasileiro de Ictiologia. Maringá, Editora da UEM. p.107-120.

Agostinho, A. A.; Júlio JR., H. F. \& Borghetti, J. R. 1992. Considerações sobre os impactos dos represamentos na ictiofauna e medidas para sua atenuação. Um estudo de caso: reservatório de Itaipu. Revista UNIMAR 14 (suplemento):89-107.

Agostinho, A. A.; Miranda, L. E.; Bini, L. M.; Gomes, L. C.; Thomaz, S. M. \& Suzuki, H. I. 1999. Patterns of colonization in neotropical reservoir, and prognoses on aging. In: TUNDISI, J. G. \& Straskraba, M. eds. Theoretical reservoir ecology and its applications. São Carlos, Brazilian Academic of Science and Backhuy. 592p.

Albrecht, M. P. \& Pellegrini-Caramaschi, E. 2003. Feeding ecology of Leporinus taeniofasciatus (Characiformes: Anostomidae) before and after installation of a hydroeletric plant in the upper rio Tocantins, Brazil. Neotropical Ichthyology 1(1):53-60.

Albrecht, M. P. \& Caramaschi, E. P. 2003. Feeding Ecology of Leporinus friderici (Teleostei, Anostomidae) in the upper Tocantins river, central Brazil, before and after installation of a hydroelectric plan. Studies on Neotropical Fauna and Environment 38:33-40.

Alvim, M. C. C. I. \& Peret, A. C. 2004. Food resources sustaining the fish fauna in a section of the upper São Francisco River in Três Marias, MG, Brazil. Revista Brasileira de Biologia 64(2): 195-202.

Araújo-Lima, C. A. R. M.; Agostinho, A. A. \& Fabré, N. N. 1995. Trophic Aspects of Fish Communities in Brazilian Rivers and Reservoirs. In: Tundisi, J. G.; Bicudo, C. E. M. \& MatsumuraTundisI, T. eds. Limnology in Brazil. Rio de Janeiro, ABC/ SBL. p.105-136.

Braga, F. M. S. B. 1990. Aspectos da reprodução e alimentação de peixes comuns em um trecho do rio Tocantins entre Imperatriz e Estreito, estados do Maranhão e Tocantins, Brasil. Revista Brasileira de Biologia 50(3):547-558.

Brandimarte, A. L.; Anaya, M. \& Shimizu, G. Y. 1999. Comunidades de invertebrados bentônicos nas fases pré e pós enchimento em reservatórios: um estudo de caso no reservatório de aproveitamento múltiplo do rio Mogi-Guaçu (SP). In: Henry, $\mathrm{R}$. ed. Ecologia de reservatórios: estrutura, função e aspectos sociais. Botucatu, Fapesp/Fundibio. p.375-408.

Casatti, L. 2001. Taxonomia do gênero sul-americano Pachyurus Agassiz, 1831 (Teleostei: Perciformes: Sciaenidae) e descrição de duas novas espécies. Comunicações do Museu de Ciência e Tecnologia PUCRS 14(2):133-178.

2002. Petilipinnis, a new genus for Corvina grunniens Jardine, 1943, (Perciformes, Sciaenidae) from the Amazon and Essequibo river basins and redescription of Petilipinnis Grunniens. Papéis Avulsos de Zoologia 42(7):169-181.

2003. Family Sciaenidae. In: Reis, R. E., Kullander, S. O. \& Ferraris, JR. C. J. eds. Check List of the Freshwater Fishes of South and Central America. Porto Alegre, EDIPUCRS. 599-602.

Chaо, L. N. 1986. A synopsis on zoogeography of the Sciaenidae. In: Uyeno, T., Arai, R., Taniuchi, T. \& Matsunra, K. eds. Indo-Pacific Fish Biology. Proceedings of the Second Intermational Conference of Indo-Pacific Fishes. Tokyo, Ichthyological Society of Japan. p.570-589.

De Filippo, R.; Gomes, E. L.; Lens-César, J.; Soares, C. B. P. \& Menezes, C. F. S. 1999. As alterações da qualidade da água durante o enchimento do reservatório de UHE Serra da Mesa 
- GO. In: Henry, R. ed. Ecologia de reservatórios: estrutura, função e aspectos sociais. Botucatu, Fapesp/ Fundibio. p.323-345.

Dumnicka, E. 1996. Upstream-downstream movement of macrofauna (with special reference to oligochaetes) in the River Raba below a reservoir. Hydrobiologia 334(3):193-198.

Hahn, N. S.; Agostinho, A. A.; Gomes, L. C. \& Bini, L. M. 1998. Estrutura trófica da ictiofauna do reservatório de Itaipu (Paraná-Brasil) nos primeiros anos de sua formação. Interciência 23(5):299-305.

Hahn, N. S.; Fugi, R.; Almeida, V. L. L.; Russo, M. R. \& Loureiro, V. E. 1997. Dieta e atividade alimentar de peixes do reservatório de Segredo. In: Agostinho, A. A. \& Gomes, L. C. eds. Reservatório de Segredo: bases ecológicas para o manejo. Maringá, EDUEM. p.141-162.

HynEs, H. B. N. 1950. The food of freshwater sticklebacks (Gasterosteus aculeatus and Pygosteus pungitius) with a review of methods used in studies of the food of fishes. Journal of Animal Ecology 19:36-58.

Hyslop, E. J. 1980. Stomach contents analysis - a review of methods and their application. Journal of Fish Biology 17:411-29.

KaWAKami, E. \& Vazzoler, G. 1980. Método gráfico e estimativa de índice alimentar aplicado no estudo de alimentação de peixes. Boletim do Instituto Oceanográfico de São Paulo 29(2):205-207.

KIKUCHI, R. M. \& UIEDA, V. S. 1998. Composição da comunidade de invertebrados de um ambiente lótico tropical e sua variação espacial e temporal. In: Nessimian, J. L. \& Carvalho, E. eds. Ecologia de Insetos Aquáticos. Série Oecologia Brasiliensis, v. V. Rio de Janeiro, Universidade Federal do Rio de Janeiro. p.157-173.
Krebs, C. J. 1989. Ecological Methodology. New York, Harper Collins. 654p.

LeCren, E. D. 1951. The lenght-weight relationship and seasonal cycle in gonad weight and condition in the perch Perca fluviatilis. Journal of Animal Ecology 20:201-219.

Magurran, A. E. 1988. Ecological Diversity and its Measurements. Princeton, Princeton University. 179p.

Margalef, R. 1983. Limnología. Barcelona, Omega. 1010p.

McCafferty, P. W. 1998. Aquatic Entomology: the Fishermen's and Ecologists' Illustrated Guide to Insects and their Relatives. Boston, Jones \& Bartlett. 448p.

Mérona, B.; Santos, G. M. \& Almeida, R. G. 2001. Short term effects of Tucuruí Dam (Amazonia, Brazil) on the trophic organization of fish communities. Environmental Biology of Fishes 60:375-392.

NikOLSKI, G. V. 1963. The Ecology of Fishes. London, Academic. 352p.

Pereira, S. M. \& DA-Silva, E. R. 1991. Descrição de uma nova espécie de Campsurus Eaton 1868 do sudeste do Brasil, como notas biológicas (Ephemeroptera: Polymitarcyidae: Campsurinae). Revista Brasileira de Biologia 51(2):321-326.

Ross, S. T. 1986. Resource partitioning in fish assemblages: a review of field studies. Copeia 2:352-138.

Strixino, G. \& Trivino-Strixino, S. 1998. Povoamento de Chironomidae em lagos artificiais. In: Nessimian, J. L. \& Carvalho, A. L. eds. Ecologia de Insetos Aquáticos. Oecologia Brasiliensis V. Rio de Janeiro, Universidade Federal do Rio de Janeiro. p.141-154.

Winemiller, K. O. 1989. Ontogenetic diet shifts and resource partitioning among piscivorous fishes in the Venezuelan Ilanos. Environmental Biology of Fishes 26:177-199.

ZAR, J. H. 1984. Biostatistical analysis. Prentice Hall, New Jersey. 718p.

Recebido em fevereiro de 2006. Aceito em maio de 2007. ISSN 0073-4721

Artigo disponível em: www.scielo.br/isz 